\title{
A Minimalist Control Strategy for Small UAVs
}

\author{
Severin Leven, Jean-Christophe Zufferey, Dario Floreano \\ Ecole Polytechnique Fédérale de Lausanne (EPFL) \\ Laboratory of Intelligent Systems (LIS, http://lis.epfl.ch) \\ CH-1015 Lausanne, Switzerland \\ \{severin.leven, jean-christophe.zufferey, dario.floreano\}@epfl.ch
}

\begin{abstract}
Most autopilots of existing Miniature Unmanned Air Vehicles (MUAVs) rely on control architectures that typically use a large number of sensors (gyros, accelerometers, magnetometers, GPS) and a computationally demanding estimation of flight states. As a consequence, they tend to be complex, require a significant amount of processing power and are usually expensive. Many research projects that aim at experiments with one, or even several, MUAVs would benefit from a simpler, potentially smaller, lighter and less expensive autopilot for their flying platforms.

In this paper, we present a minimalist control strategy for fixed-wing MUAVs that provides the three basic functionalities of airspeed, altitude and heading turnrate control while only using two pressure sensors and a single-axis rate gyro. To achieve this, we use reactive control loops, which rely on direct feedback from the sensors instead of full state information. In order to characterize the control strategy, it was implemented on a custom-made autopilot. With data recorded during flight experiments, we carried out a statistical analysis of step responses to altitude and turnrate commands as well as responses to artificial perturbations.
\end{abstract}

\section{INTRODUCTION}

Aerial robotic platforms able to fly autonomously in an outdoor environment attract an increasing number of researchers because they are useful tools for a large range of experiments as well as for education. Examples include obstacle avoidance [1], testing of new sensors [2], flight control or navigation strategies, observation tasks [3] and gathering of air data, e.g. pollution or toxic plumes [4]. In the past decade, an additional research interest for collective and networked aerial systems has emerged [4], [5].

A major drawback of existing MUAV autopilots is their costly and complex design. The most common implementation of flight control [6] (Fig. 1, top), inspired from classical aircraft control architectures [7]-[8], involves a multitude of sensors and a full estimation of the flight states (position, orientation and velocity). In many MUAVs [9]-[12], a complex and computationally expensive Attitude and Heading Reference System (AHRS) combined with a GPS estimates all aircaft states that cannot be measured directly using complementary or Bayesian filters such as a Kalman filter [11], [13]. Examples of autopilots using full state estimation are the commercial products Procerus Kestrel ${ }^{\mathrm{TM}}$, Cloudcap Piccolo and the MicroPilot ${ }^{\circledR}$ MP series. Their built-in AHRS computes the aircraft's attitude angles, fusing sensor data of orthogonally mounted rate gyros, accelerometers and magnetic sensors (three of each). For airspeed and altitude

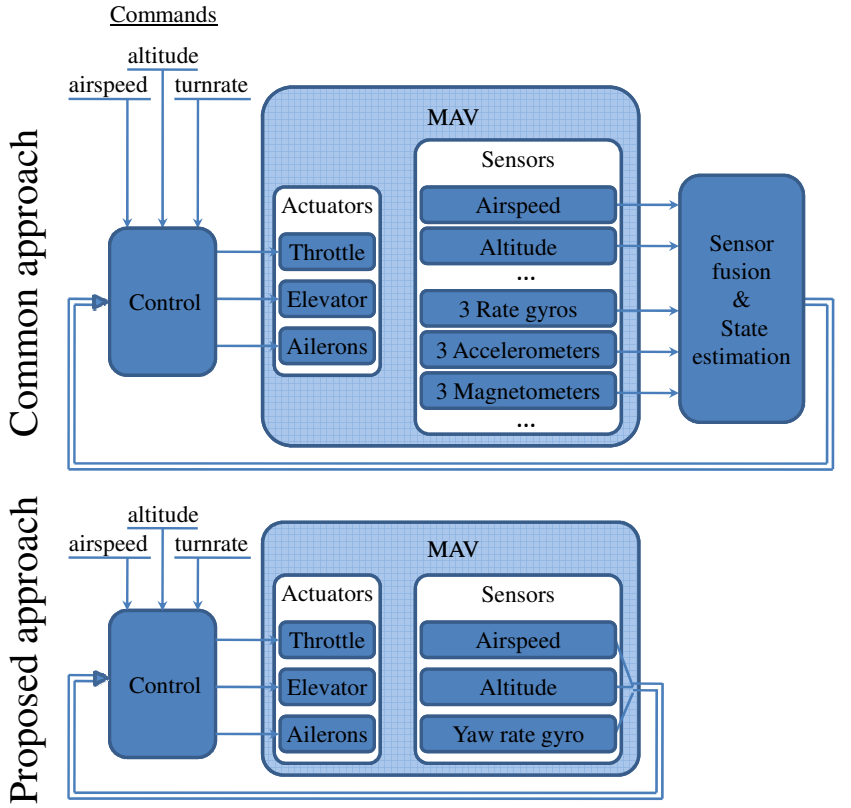

Fig. 1. Comparison between the common MUAV control strategy and the one proposed in this paper for the three basic control functionalities: airspeed, altitude and heading turnrate control. In our case, the sensor fusion and state estimation block is eliminated, and the number of sensors is reduced to a minimum.

control, additional sensors have to provide appropriate measurements (usually pressure transducers or GPS).

In this paper, we take an alternative approach to flight control and present the design of a minimalist control strategy for fixed-wing MUAVs that performs control of airspeed, altitude and heading turnrate (not to be confused with the aircraft's yaw rate). This allows to have the same set of control commands as a 2D wheeled robot that can additionally modify its altitude. Our goal is to enable easy programming of behaviors by other users, e.g. researchers, on top of the proposed strategy. For instance, this could be higher level controllers using a behavior-based approach [5] or waypoint navigation [14].

Unlike the autopilots described above, our approach aims at reducing the number of sensors and eliminating the complex flight state estimation process in order to eventually obtain lighter, smaller and lower cost autopilots. Our control strategy (Fig. 1, bottom) indeed uses only three sensors as control inputs: an airspeed and an altitude sensor in addition 
to a single-axis rate gyro.

Of course, eliminating state feedback may lead to accuracy and stability problems, e.g. due to the fact that damping and frequency of the platform's eigen-dynamics cannot be modified freely and independently. Furthermore, it may not be possible to assess critical aircraft states, such as the stall pitch angle, if no estimate of the pitch attitude is available. Although these issues are critical for large, manned aircraft, they are less so for MUAVs, especially when the flight envelope is limited to normal flight. By normal flight, which we assume for the development of our minimalist control strategy, we understand non-inverted flight (roll angles between $\pm 90^{\circ}$ ) at a platform's typical cruise speed and on slopes with max. inclinations of $\pm 20^{\circ}$ (and similar pitch angle values). In order to perform a characterization of the proposed method, we did not go through the lengthy process of modelling, parameter identification and finally an analytical or numerical stability analysis. Instead, we show by means of a systematic set of flight experiments that the control system operates robustly and maintains a good level of performance in terms of control errors and repeatability.

\section{CONTROL STRATEGY}

A minimalist control strategy that can perform airspeed, altitude and heading turnrate control is depicted in Fig. 1, bottom.

To avoid state estimation through a sensor fusion process, sensors should ideally provide direct input for control. Airspeed and altitude can be measured with a single sensor each, such as absolute or differential pressure sensors, but no simple sensor exists to measure the heading turnrate. A yaw rate sensor or a magnetic compass cannot be used alone to determine the heading turnrate because of the non-zero bank (roll) angle during turns. In order to find a solution to this sensing problem and to preserve the idea of using a minimal set of sensors, we propose to make assumptions on flight dynamics during turns:

\section{A. Prerequisites}

We assume that all turn maneuvers can be considered as coordinated turns. The basic definition of a coordinated turn given in [7] is an idealized turn maneuver at constant flight speed during which all components of force along the aircraft's lateral axis sum to zero. In the following, we will use the expression coordinated turns not only in the above sense, but include the assumptions of level flight, zero sideslip angle and small angle of attack.

The zero sideslip condition is usually met by coordinating rudder and aileron deflections. Most aircraft that do not have an active rudder (such as flying wings) are designed to have a natural tendency to reduce the sideslip angle, e.g. due to a delta/arrow wing shape or passive rudder surfaces. The small angle of attack condition is an approximation, but generally well met (values are typically $<15^{\circ}$ ).

Knowledge of the theoretical dynamics during such turns allows to find a solution to the heading turnrate sensing problem: first, it enables us to transform heading turnrate



Fig. 2. An aircraft executing a coordinated, level turn. The yaw rate $r$ and pitch rate $q$ are constant. The forces $F_{L}$ (Lift), $F_{G}$ (Gravity) and $F_{C}$ (Centrifugal) are shown as functions of the vehicle mass $m$, airspeed $v$, gravity $g$, bank angle $\Phi$ and heading turnrate $\dot{\Psi}$.

control into bank angle control. Second, it provides an easy way to obtain a bank angle estimate that does not even require any computationally expensive sensor fusion.

When using the assumption of coordinated turns as defined above, we can take advantage of the kinematic and dynamic relationships between the involved variables (Fig. 2), in order to derive a relationship between the heading turnrate $\dot{\Psi}$ and the bank angle $\Phi$.

Based on the equation for lateral equilibrium of the acting forces, we find

$$
\begin{aligned}
F_{C} & =F_{L} \sin (\Phi) \\
\Rightarrow m \dot{\Psi} v & =m g \tan (\Phi)
\end{aligned}
$$

where $F_{L}$ denotes the lift force, $F_{C}$ the centrifugal force, $v$ the measured flight speed and the $g$ gravity acceleration. A given heading turnrate command $\dot{\Psi}_{c}$ consequently translates into a bank angle command $\Phi_{c}$ as follows (the airspeed $v$ is assumed to be known):

$$
\Phi_{c}=\arctan \left(\frac{v}{g} \dot{\Psi}_{c}\right)
$$

This means that if in addition to (3) a measurement of the bank angle $\Phi$ is available, heading turnrate control can be formulated in terms of the bank angle $\Phi$. Then, it is not necessary to measure the heading turnrate.

Given the assumption of coordinated turns, the roll angle $\Phi$ can indeed be computed directly from current yaw rate and airspeed measurements. The yaw rate $r$ can be expressed as a function of heading turnrate and roll angle as follows

$$
r=\dot{\Psi} \cos (\Phi)
$$

Combining (2) and (4) yields the following simple relationship between the roll angle $\Phi$ and the yaw rate $r$ (measurable with a yaw rate gyro):

$$
\Phi=\arcsin \left(\frac{v}{g} r\right)
$$






Fig. 3. Complete view of the controller structure including airspeed, altitude and heading turn control based on 3 sensor inputs, 3 external control commands and 3 actuator outputs. Neither complex sensor-fusion, extensive filtering and attitude integration, nor nested control loops are used, which creates very short and reactive signal pathways between sensor feedback and actuators.

However, using the arcsin-function in (5) is critical since it is highly non-linear for increasing arguments. Therefore, sensor noise on $r$ and $v$ is amplified non-linearly as well, and the argument of the arcsin-function may even have values exceeding the maximum allowed input value of \pm 1 . A possible remedy is to use an approximation function with a graceful behavior for increasing values of $r$ and $v$. We chose an approximation that is based on a partial Taylor linearization around $r=r_{0}$ and that scales non-linearly with the $\frac{v}{g}$-term:

$$
\begin{aligned}
\Phi & \left.\approx \frac{\delta}{\delta r} \arcsin \left(r \frac{v}{g}\right)\right|_{r=r_{0}} \cdot\left(r-r_{0}\right) \\
& \approx \frac{v}{g} \cdot \frac{1}{\sqrt{1-\left(r_{0} \frac{v}{g}\right)^{2}}} \cdot r
\end{aligned}
$$

In order to have $\Phi=0^{\circ}$ for $r=0^{\circ} / s$, we obtain (7) from (6) through an affine transformation. Finally, we chose $r_{0}=22.5^{\circ} \mathrm{s}$ such that the approximation fits the arcsinfunction well $\left(< \pm 1^{\circ}\right)$ for bank angles up to about $45^{\circ}$. The maximum airspeed allowed for the approximation is therefore determined by

$$
\left(r_{0} \frac{v_{\max }}{g}\right)^{2}=1
$$

to $v_{\max } \approx 25 \mathrm{~m} / \mathrm{s}$, sufficient for MUAVs.

With these notions at hand, we describe in the next paragraphs the elements of our minimalist controller. A schematic of the full controller structure is depicted in Fig. 3.

\section{B. Airspeed Control}

For airspeed control, a single loop containing a PI controller is enough to minimize control errors by means of acting on the throttle actuator $\delta_{t}$. The integral controller term ensures a correct throttle trim value and eliminates static errors.

\section{Altitude Control}

For altitude control, our control strategy consists of two components: a PID controller and a bank compensator. The bank compensator has been implemented to assist the PID altitude controller during steep turns, where it generates the necessary additional lift and therefore limits altitude variations. Both components act directly on the elevator $\delta_{e}$.

A major difference with respect to common autopilots (e.g. [7]) is the absence of nested control loops for pitch rate and pitch attitude. Our results show that neither of these are necessary for our experimental flying platform: intrinsic stability of the pitch-eigendynamics (damping) and a limitation of the elevator deflection suffice for longitudinal flight stability. Limiting the elevator deflection is equivalent to limiting the climb and descent rate for transitions between commanded altitudes. However, other platforms with less intrinsic stability in the pitch axis may require an additional pitch damper and a pitch rate gyro.

In order to dampen altitude oscillations (phugoideigendynamics, [7]) and fast transitions, we rely on the derivative term of the altitude controller as a substitute for the unavailable pitch angle $\Theta$. The integral controller term ensures a correct elevator trim value and cancels static errors.

Since the lift vector tilts from the vertical axis according to the bank angle (Fig. 2), more lift is needed during turns for level flight. The increase in lift $\Delta F_{L}$ depending on the bank angle $\Phi$ is given by

$$
\Delta F_{L}=m g\left(\frac{1}{\cos (\Phi)}-1\right)
$$

Assuming that a linear increase of the elevator angle $\delta_{e}$ leads to a linear change in lift $\Delta F_{L}$ by a factor $k$, an apriori, open-loop command of the elevator $\delta_{e-c o m p}$ (the bank compensator) can be tuned with

$$
\frac{\Delta F_{L}}{k}=\delta_{e-\operatorname{comp}}=\frac{m g}{k}\left(\frac{1}{\cos (\Phi)}-1\right) \approx \frac{m g}{2 k} \Phi^{2}
$$

The $\frac{1}{\cos }$-function in (10) diverges quickly for increasing values of $\Phi$. It is replaced by a first-order Taylor-series approximation in order to limit sensitivity to sensor noise in the $\Phi$-estimate. Theoretical equation and approximation match again well for roll angles up to $\Phi=45^{\circ}$. 



Fig. 4. Left side: The flying-wing aircraft $(80 \mathrm{~cm}$ wingspan, $350 \mathrm{~g}, 30 \mathrm{~min}$ endurance) used to test the proposed control strategy. Right side: Customdesigned flight controller including 2 pressure sensors, the yaw rate gyro as well as a DC-DC power supply circuitry and connectors to standard R/C servos. The board measures $42 \times 32 \times 14 \mathrm{~mm}$ and weighs $14.9 \mathrm{~g}$.

\section{Turn Control}

Using the results of section II-A, the turn controller has the only task of minimizing the error between the commanded bank angle $\Phi_{c}(3)$ and the estimated one (7). To achieve this, a PD controller calculates the aileron output $\delta_{a}$ based on the control error. The derivative term dampens the roll motion during fast bank angle transitions.

\section{EXPERIMENTAL SETUP AND CHARACTERIZATION METHODS}

The experimental setup on which the presented flight control strategy is tested consists of the following 4 components:

- A flying wing platform [15] controlled by elevons (combined ailerons/elevator) and thrust of a propellor driven by an electric motor (Fig. 4, left).

- Custom flight control electronics, built around a dsPic33 micro-controller (Fig. 4, right). The measurements of airspeed and altitude are provided by two pressure sensors (Freescale MPX series), the yaw rate by a rate gyroscope (Analog Devices ADXRS610). Speed, altitude and turnrate commands can be issued by an external onboard processing unit or through a wireless link with a ground station. Standard R/C equipment is used for actuators, motor controller and battery.

- A monitoring software which displays and logs flight data transferred via a wireless link (MaxStream XBee$\mathrm{PRO}^{\mathrm{TM}}$ ) in real-time to a ground station, using the "Ishtar" open-source software [16].

- An XSens MTi-G AHRS linked to a uBlox LEA-5H GPS receiver, which is not used for control but as ground truth (position and attitude) for the characterization of our own approach.

In order to avoid the lengthy procedure of aircraft modelling and optimization of flight controller parameters, the few parameters of the proposed control strategy are adjusted manually in flight experiments, with intuitively estimated initial values. The clear organization of the controller into airspeed, altitude and turnrate allows a step-by-step tuning procedure, whereby aircraft control is progressively passed over from a human operator to the autopilot. The first step

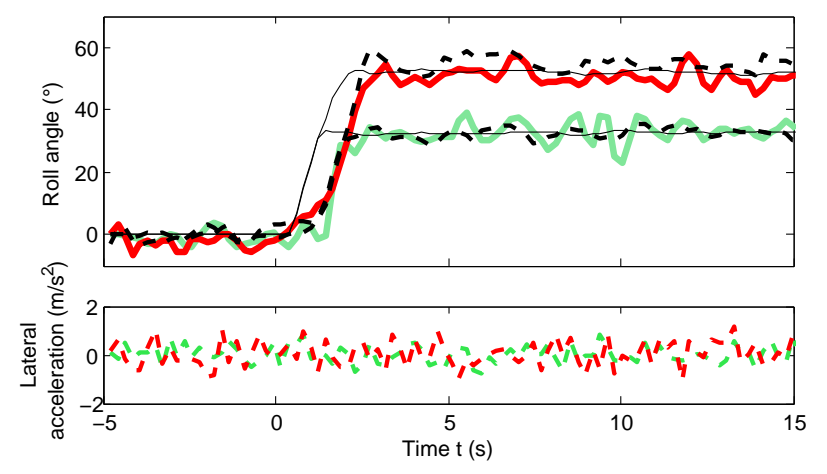

Fig. 5. Validity of the turn coordination assumption with the chosen platform for heading turnrate steps of $30 \%$ and $60 \% \mathrm{~s}$ at an airspeed of about $12 \mathrm{~m} / \mathrm{s}$. Roll rate commands (thin lines) were computed onboard the flying platform based on (3). XSens MTi-G AHRS readings are shown as dashed lines. Acceleration shown is in lateral aircraft axis.

consists in tuning the airspeed controller while altitude and turnrate are under manual control. The second step is to tune the altitude controller while keeping the aircraft manually in straight flight or slighty banked turns. Then, the bank compensator is adjusted during turns with high bank angles with the goal of maintaining level flight. The final step is to set up the turnrate controller parameters to a compromise of fast response time and stability margin. The integral terms of airspeed and altitude controller are chosen small, to act as long-term trim values. No particular difficulties were encoutered in the parameter tuning process. As operating point for typical flights we chose $50 \mathrm{~m}$ height $(\approx 450 \mathrm{~m}$ absolute altitude) and $12 \mathrm{~m} / \mathrm{s}$ airspeed.

In order to characterize the proposed control strategy, we first carried out several series of flight experiments during which steps in altitude or heading turnrate commands as well as perturbations from steady-state flight conditions were triggered manually. We also investigated combined turn-andclimb maneuvers during which the assumptions of level, coordinated turns are not respected. Data of 20 trials for each experiment were collected from at least two flights on two different days, each with stable atmospheric conditions and windspeeds up to $5 \mathrm{~m} / \mathrm{s}$. A statistical analysis of the recorded flight data allows us to make statements about control performance (repeatability, accuracy, transition speeds) and flight stability. For all experiments, flight data were compared to the ground truth provided by the XSens MTi-G.

\section{RESULTS}

Before characterizing the implementation of our control strategy, we verified the assumption of coordinated turns (i.e. the condition of zero acceleration along the aircraft's lateral axis) and check that the roll angle $\Phi$ can indeed be estimated with our method according to (7). To this end, we performed a series of flights with all controllers (airspeed, altitude and turnrate) tuned and engaged. Recorded data of two heading turnrate steps $\left(30^{\circ} \mathrm{s}\right.$ and $\left.60^{\circ} / \mathrm{s}\right)$ during level flight are shown in Fig. 5: the roll angle estimate, the XSens AHRS' roll angle and the roll angle command from (3) match well, 
a) Altitude step
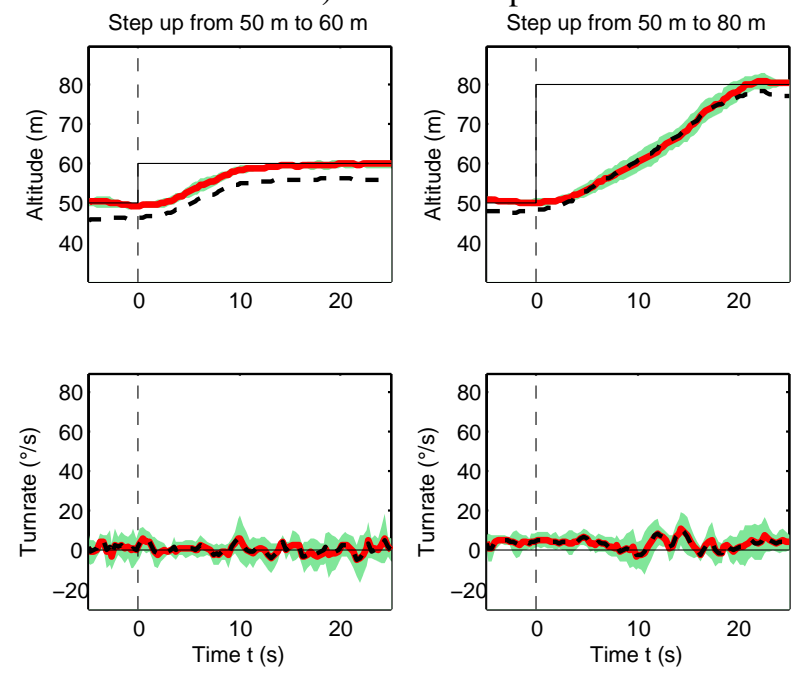

c) Altitude and heading turnrate step


b) Heading turnrate step
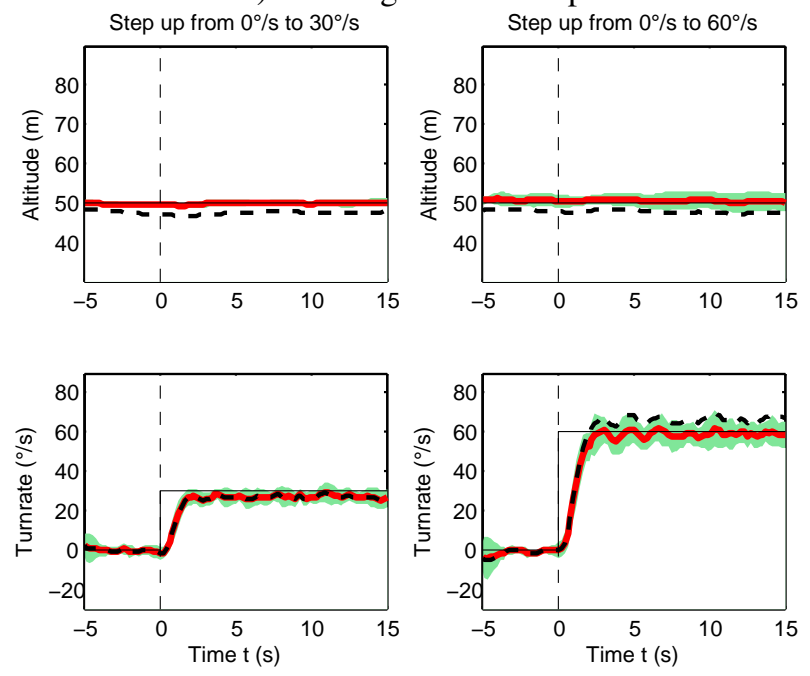

d) Reaction to elevator/aileron perturbations


Fig. 6. Snapshots of the system's (MUAV platform and implemented control strategy) reaction to (a) closed-loop altitude command steps, (b) turnrate command steps and (c) combined altitude and turnrate command steps (c). In (d), the grey zone indicates that a fixed elevator/aileron deflection is applied while the controller is shut off. Shown are the command steps (thin lines) at time $t=0$, the mean trajectories (bold line) and the standard deviation (as surrounding area) of 20 flights in each experiment. Mean XSens MTi-G readings are shown as dashed lines.

even for bank angles exceeding $45^{\circ}$. The additonal fact that the lateral acceleration has no noticeable offset during turns confirms the intrinsic tendency of the chosen flying platform for coordinated turns.

As a next step, we characterized the controller performance. Statistical results from the experiments, displaying the mean trajectories and the standard deviation for the 20 trials per experiment, are shown for altitude steps (Fig. 6a), for heading turnrate steps (Fig. 6b) and for steps in both altitude and turnrate simultaneously (Fig. 6c). In order to demonstrate the controller's ability to stabilize the aircraft from perturbations, short elevator/aileron deflections were commanded while the controller was temporarily switched off (Fig. 6d). During all experiments, the airspeed controller maintained airspeed constant at $12 \mathrm{~m} / \mathrm{s} \pm 1 \mathrm{~m} / \mathrm{s}$ (standard deviation). The barometric pressure sensor provided the altitude measurement, the heading turnrate was obtained according to (4) using the yaw rate and the $\Phi$-estimate.

The experiments with altitude steps (Fig. 6a) show a small standard deviation, meaning good repeatability of the trajectories. When compared to the ground truth of the reference sensor, we can see that tracking of a desired altitude command is achieved with an accuracy of a few meters. While the slow drift of the altitude measurement of the barometric pressure sensor due to temperature and atmospheric changes is visible in the plots, it does not influence short-term control performance. During altitude transitions, the mean turnrate remains close to $0 \% \mathrm{~s}$ with short-term peaks never exceeding $\pm 10^{\circ} \mathrm{s}$. This is small enough to be considered as straight flight. After issuing the command step, a short delay 
of about $1 \mathrm{~s}$ passes before a reaction of the system (MUAV platform including the control system) is noticeable. The climb rate of about $1.5 \mathrm{~m} / \mathrm{s}$ is limited by elevator saturation settings in the altitude controller.

The experiments with turnrate steps show an immediate reaction of the system. After about $1 \mathrm{~s}$ rise time the commanded turnrate (Fig. 6b) is reached. Thanks to the bank compensator (10), no drop in altitude occurs. From the experiment with $60^{\circ} \mathrm{s}$ heading turnrate, which corresponds to more than $45^{\circ}$ bank angle (3), the effect of slightly underestimating the turnrate, resp. the bank angle with the linear approximation function (7) can be noticed, without affecting control stability. Otherwise, correspondance between estimation and the XSens AHRS for lower turnrates is excellent.

In order to show that the assumption of coordinated turns does not limit turn maneuvers to strictly level flight, experiments with a simultaneous step of both altitude and turnrate commands have been done as well. Fig. 6c shows that the control task is still successfully performed.

The goal of our last experiment was to test whether the controller is able to cope with short but significant perturbations (a fixed elevator/aileron deflection was applied while the controller was disabled). The plots in Fig. 6d show that perturbations on the elevator lead to fast $10 \mathrm{~m}$ climbs, perturbations on the ailerons to heading turnrates of $70 \% \mathrm{~s}$, and that the controller easily stabilizes the aircraft as soon as it is enabled again. For the elevator perturbations, the absence of a pitch rate control loop does not seem to be problematic.

\section{CONCLUSION}

We presented a minimalist control strategy for fixed-wing MUAVs that is different from typical current autopilots in that it only relies on two pressure sensors and a single axis rate gyro. It takes advantage of theoretical considerations on flight dynamics in so-called coordinated turns, which allows to eliminate the need for sensor fusion and complex state estimation to perform airspeed, altitude and heading turnrate control. These are the required basic functionalities on top of which behavior-based or waypoint-navigationbased controllers can conveniently be implemented. With statistical results from flight experiments with a small flyingwing type platform we have demonstrated the viability and good performance of the proposed control strategy.

Unlike infrared sensors or GPS used for the same control tasks in other minimalist control solutions [17]-[18], the proposed set of simple sensors has no need for calibration and works in all environment conditions.

Reducing the number of sensors and eliminating state feedback may potentially lead to stability problems. However, this was not observed during our experiments thanks to the intrinsic static and dynamic stability of our test platform.

A detailed assessment of how critical the absence of attitude information is for flight stability could still be useful to do, especially for platforms with less intrinsic stability.

Another aspect of future work will be to set up automatic parameter tuning procedures, which will benefit fully of the simple controller structure and the very restricted number of parameters involved in the control loops.

\section{ACKNOWLEDGEMENTS}

The authors wish to thank Sabine Hauert who helped with flight experiments and provided feedback on the autopilot in the framework of the Swarming Micro Air Vehicles project (http://lis.epfl.ch/smavs), a first application [5] for the proposed minimalist control stategy. This work is supported by armasuisse, competence sector Science + Technology for the Swiss Federal Department of Defence, Civil Protection and Sports.

\section{REFERENCES}

[1] Z. He, R. V. Iyer, and P. R. Chandler, "Vision-based UAV Flight Control and Obstacle Avoidance," in Proc. of the IEEE American Control Conference, Minneapolis, Minnesota, June 2006.

[2] J. Saunders, B. Call, R. Beard, and T. McLain, "Static and Dynamic Obstacle Avoidance for Miniature Air Vehicles," in Proc. of the AIAA Infotech@Aerospace Conference, Washington, DC, Sept. 2005.

[3] N. Nigam and I. Kroo, "Persistent Surveillance Using Multiple Unmanned Air Vehicles," in Proc. of the IEEE Aerospace Conference, Big Sky, Montana, March 2008.

[4] J. Allred, A. B. Hasan, and W. Pisano, et al., "SensorFlock: An Airborne Wireless Sensor Network of Micro-Air Vehicles," in Proc. of the $5^{\text {th }}$ Int. Conference on Embedded Networked Sensor Systems (SenSys'07), Sydney, Australia, November 2007.

[5] S. Hauert, J.-C. Zufferey, and D. Floreano, "Swarming without positioning information: an application in aerial radio communication relay," to appear in: Autonomous Robots, 2008.

[6] H. Chao, Y. Cao, and Y. Chen, "Automation of Small UAVs Using a Low Cost MEMS Sensor and Embedded Computing Platform," in Proc. of the IEEE International Conference on Mechatronics and Automation, Harbin, China, Aug. 2007.

[7] B. L. Stevens and F. L. Lewis, Aircraft Control and Simulation, 2nd ed. Wiley, Oct. 2003

[8] B. Etkin and L. D. Reid, Dynamics of Flight: Stability and Control, 3rd ed. Wiley, Oct. 1995.

[9] J. S. Jang and C. G. Tomlin, "Autopilot Design for the Stanford DragonFly UAV: Validation through Hardware-in-the-Loop Simulation," in Proc. of the AIAA Guidance, Navigation and Control Conference (GNC), Montreal, Aug 2001.

[10] J.-H. Kim, S. Wishart, and S. Sukkarieh, "Real-time Navigation, and Control of a UAV using Low-cost Sensors," in Proc. of the International Conference on Field And Service Robotics (FSR 2003), Yamanashi, Japan, July 2003, pp. 95-100.

[11] D. B. Kingston and R. W. Beard, "Real-Time Attitude and Position Estimation for Small UAVs Using Low-Cost Sensors," in Proc. of the AIAA $3^{\text {rd }}$ Unmanned Unlimited Systems Conference and Workshop, Chicago, September 2004.

[12] R. Beard, D. Kingston, M. Quigley, D. Snyder, R. Christiansen, and W. Johnson, "Autonomous vehicle technologies for small fixedwing UAVs," Journal of Aerospace Computing, Information, and Communication, vol. 2, no. 1, pp. 92-108, 2005.

[13] M. Niculescu, "Sensor Fusion Algorithms for Unmanned Air Vehicles," in Proc. of the IEEE Information, Decision and Control Symposium, Adelaide, Australia, Feb. 2002.

[14] J. J. Kehoe, R. Causey, M. Abdulrahim, R. Lind, J. W. Grzywna, J. Plew, and M. C. Nechyba, "Waypoint Navigation for a Micro Air Vehicle using Vision-Based Attitude Estimation," in Proc. of the 4th European Micro Air Vehicle Conference (EMAV), Braunschweig, July 2004.

[15] S. Leven, J.-C. Zufferey, and D. Floreano, "A Simple and Robust Fixed-Wing Platform for Outdoor Flying Robot Experiments," in Proc. of the $1^{\text {st }}$ Int. Symposium on Flying Insects and Robots (FIR), Monte Verità, Locarno, Switzerland, August 2007, pp. 69-70.

[16] A. Beyeler, S. Magnenat, and A. Habersaat, "Ishtar: a Flexible and Light-Weight Software for Remote Data Access," in Proc. of the $4^{\text {th }}$ European Micro Air Vehicle Conference and Competition (EMAV'08), Braunschweig, Germany, July 2008.

[17] P. Brisset, A. Drouin, and M. Gorraz, "The Paparazzi Solution," in Proc. of the $2^{\text {nd }}$ Micro Air Vehicle Conference and Competition (EMAV'06), Braunschweig, Germany, July 2006.

[18] W. J. Pisano and D. A. Lawrence, "Autonomous UAV Control Using a 3-Sensor Autopilot," in Proc. of the AIAA Infotech@Aerospace Conference and Exhibit, Rhonert Park, California, May 2007. 